\title{
Cinq étapes à suivre afin de créer un site Web parfait pour votre cabinet
}

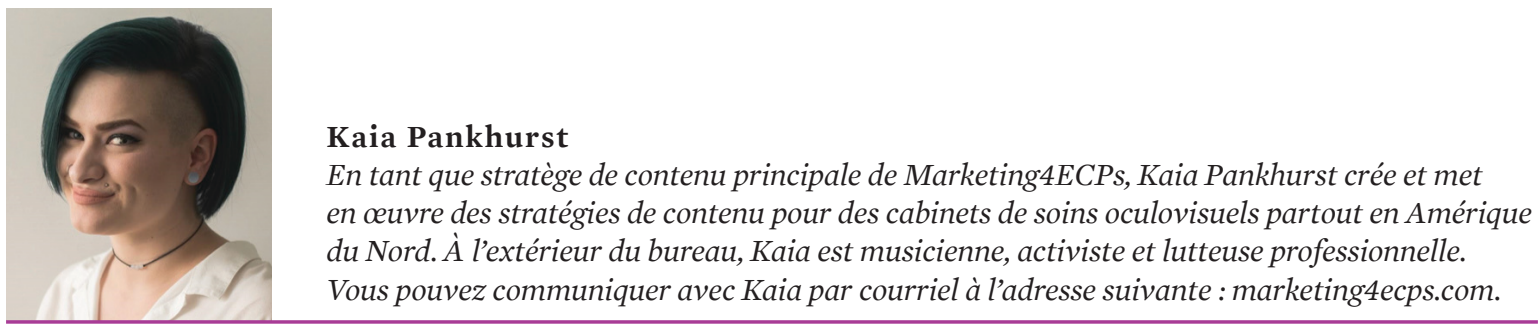

$\mathrm{N}$

ous vivons dans un monde numérique, alors un site Web est essentiel si vous voulez que votre cabinet se démarque. Toutefois, il ne suffit pas d'avoir seulement un site Web; si vous voulez concurrencer les autres cabinets en ville (surtout les magasins à grande surface), vous avez besoin d'un excellent site Web.

Idéalement, vous embaucherez une agence spécialisée dans la création de sites Web à conversion élevée et qui comprend l'industrie des soins oculovisuels. Que vous créiez un nouveau site Web ou mettiez à jour votre site actuel, ces cinq étapes pourraient être suffisantes pour faire passer votre site Web au niveau supérieur.

\section{TENEZ COMPTE DE VOTRE UTILISATEUR}

La chose la plus importante à garder à l'esprit lors de la création ou de la mise à jour du site Web de votre cabinet est l'expérience utilisateur. Vous devez en tenir compte pour presque tous les aspects de votre site Web. Par exemple, $53 \%$ des utilisateurs abandonneront une page si elle ne se charge pas en trois secondes. De plus, les utilisateurs ne lisent pas le contenu du site Web mot pour mot; ils parcourent le site. Le contenu de votre site Web devrait donc être divisé en petits paragraphes dont les titres visent à faciliter le survol.

Garder en tête les besoins en matière d'information de vos utilisateurs pour créer votre site Web est un excellent point de départ.

PRÉSENTEZ-VOUS

Marketing4ECPs a créé d'innombrables sites Web au fil des ans, et nous avons remarqué une tendance fascinante. La page que les utilisateurs consultent le plus après la page d'accueil est la page « $\grave{A}$ propos de notre cabinet ».

Les patients éventuels veulent savoir qui vous êtes et quelle est votre vision. Assurez-vous d'inclure votre énoncé de mission, vos valeurs principales et une courte biographie de vos docteurs et de tout autre membre du personnel que vous voulez mettre en valeur.

Aussi, assurez-vous de mentionner les organismes de bienfaisance ou communautaires avec lesquels vous travaillez.86 \% des consommateurs recherchent des marques axées sur des objectifs.

\section{PRÉSENTEZ VOS SERVICES}

Vous n'aurez jamais de nouveaux clients pour la sécheresse oculaire si les consommateurs de votre région ne savent pas que vous offrez des traitements spécialisés pour la sécheresse oculaire. Votre site Web doit mettre en évidence tous les services que vous offrez, ou du moins tous les principaux services que vous offrez. Il faut aussi tenir compte du fait que les patients qui consultent Internet entreront des symptômes, car ils ne savent pas nécessairement qu'ils souffrent de sécheresse oculaire. Il est donc essentiel que votre page sur la sécheresse oculaire contienne des mots-clés renvoyant aux symptômes pour qu'elle apparaisse dans les résultats de recherche. 
Idéalement, chaque service devrait avoir sa propre page Web. Cela vous donnera les meilleurs résultats en matière de référencement. Mais à tout le moins, vous devriez inclure une liste de tous vos services quelque part sur votre site Web.

\section{MODERNISEZ VOTRE MARQUE}

Les sites Web qui ont une apparence soignée et moderne donnent de bons résultats. L'investissement dans la conception de votre site Web montre aux utilisateurs que vous êtes disposé à évoluer avec le temps et en mesure de le faire. Un site Web de 2012 pourrait répondre à des besoins élémentaires, mais il pourrait donner une fausse impression aux consommateurs.

Vous n'avez pas nécessairement besoin de créer un tout nouveau logo ou de changer les couleurs de votre marque. Cependant, vous devriez vous assurer que les images sur votre site Web sont récentes et inclusives. Utilisez des polices simples et lisibles, optez pour une mise en page qui facilite la navigation et incorporez des couleurs et des textures que l'on retrouve dans votre cabinet si vous le pouvez. Avons-nous mentionné les règles de conception afin d'expliquer les raisons pour lesquelles un consommateur choisit un site Web d'optométrie?

\section{RÉPONDRE AUX QUESTIONS IMPORTANTES}

Lorsque les gens font une recherche sur Google, ils posent presque toujours une question. Quels cabinets d'optométrie sont-ils situés le plus près de chez moi? Pourquoi mes yeux sont-ils secs? À quelle fréquence ai-je besoin d'un examen de la vue?

Si votre site Web répond à ces questions, ce sera avantageux pour votre cabinet.

Vos pages de service sont un bon endroit pour répondre aux questions. Réfléchissez aux questions que vos clients vous posent le plus souvent, puis créez une section de foire aux questions (FAQ) en y intégrant ces questions (et, surtout, leurs réponses).

Certaines questions sont un peu trop précises ou spécialisées pour être pertinentes sur une page de service. Les blogues sont un excellent moyen de répondre à certaines des questions les plus complexes, comme « À quel âge un enfant peut-il commencer à porter des lentilles cornéennes? » ou «Quel type de chirurgie réfractive au laser me convient? »

Le fait de donner aux utilisateurs l'accès à votre expertise renforce la confiance, de sorte que même si l'utilisateur n'a pas besoin de prendre un rendez-vous aujourd'hui, il sera plus susceptible de faire appel à votre cabinet le moment venu.

\section{VOTRE SITE WEB EST VIVANT}

Quoi qu'il en soit, il est important de se rappeler qu’à mesure que la technologie, la science médicale et les comportements des utilisateurs changeront, les pratiques exemplaires en matière de site Web changeront également. Il est préférable de continuer à optimiser et à actualiser votre site Web pour en tirer le meilleur rendement possible.

Si vous pouvez rester au fait des tendances numériques en constante évolution, vous aurez aussi de très bonnes chances de demeurer un chef de file de votre marché. • 